\title{
Standards retrodigitaler Wörterbücher*
}

\author{
Michael Schlaefer, Deutsches Wörterbuch, \\ Akademie der Wissenschaften in Göttingen, Deutschland
}

Zusammenfassung: In den vergangenen Jahren sind zunehmend die Möglichkeiten der rückwirkenden digitalen Textaufbereitung auch für Wörterbücher genutzt worden. Für die vorliegenden deutschsprachigen Wörterbücher zu älteren Sprachstufen und für wortgeschichtliche Wörterbücher stellen sich dabei besondere Probleme. Diese Probleme betreffen nur zu einem Teil technische oder informatische Zusammenhänge. Sie ergeben sich vor allem aus dem lexikographischtechnischen und dem inhaltlichen Zustand der Wörterbücher. Wie die vorliegenden digitalen Wörterbuchbeispiele erkennen lassen, erweisen sich die Möglichkeiten, durch die Retrodigitalisierung für diese Wörterbücher neue, vor allem systematische Nutzungsmöglichkeiten zu erschließen, als teilweise sehr begrenzt. Das Verhältnis von Aufwand und Ergebnis muß im Einzelfall kritisch geprüft werden. Im Zweifelsfall muß die Erarbeitung neuer Wörterbücher den Vorrang vor einer aufwendigen kostenintensiven digitalen Aufbereitung älterer, inhaltlich überholter Werke haben.

Stichwörter: LEXIKOGRAPHIE, DIGITALES WÖRTERBUCH, HISTORISCHES WÖRTERBUCH, MITTELHOCHDEUTSCHES WÖRTERBUCH, GRIMMSCHES WÖRTERBUCH, GOETHE-WÖRTERBUCH, DEUTSCHES RECHTSWÖRTERBUCH, MIKROSTRUKTUREN, LEXIKOGRAPHISCHE METASPRACHE, WÖRTERBUCHKRITIK, ZUKÜNFTIGE WÖRTERBÜCHER

\begin{abstract}
Standards for retrodigitalised dictionaries. In recent years, text retrodigitalisation has been applied increasingly to dictionaries. Retrodigitalisation faces special problems in respect of both dictionaries of older language stages and historical dictionaries of German currently in use. There are technical problems as well as issues of computerisation that need to be addressed, but the main obstacle is the poor quality of lexicographical content and technique. Current retrodigitalised dictionaries show the limitations of retrodigitalisation, especially for systematic data retrieval. Cost-benefit analysis should be applied to each individual case. In case of doubt, the creation of new dictionaries should be preferred to the large-scale and expensive retrodigitalisation of older lexicographically antiquated works.
\end{abstract}

Keywords: LEXICOGRAPHY, DIGITAL DICTIONARY, HISTORICAL DICTIONARY, MITTELHOCHDEUTSCHES WÖRTERBUCH, GRIMMSCHES WÖRTERBUCH, GOETHE-WÖRTER-

* Der Artikel beruht auf einem gleichlautenden Kurzreferat anläßlich der 3. Arbeitstagung deutschsprachiger Akademiewörterbücher vom 22.3. - 25.3.2000 in Zürich. Für ihre Unterstützung bei der Durchsicht und Fertigstellung des Manuskripts danke ich Dr. H. Albrand, U. Härtel, D. Holste, F. Michaelis und F. M. Wohlers.

Lexikos 10 (AFRILEX-reeks/series 10: 2000): 157-172 
BUCH, DEUTSCHES RECHTSWÖRTERBUCH, MICROSTRUCTURES, LEXICOGRAPHICAL METALANGUAGE, DICTIONARY CRITICISM, FUTURE DICTIONARIES

Die nachträgliche Umsetzung von Printprodukten in das digitale Medium besitzt seit einiger Zeit eine gewisse Konjunktur, auch wenn der Anteil an retrodigitalen Wörterbüchern im Vergleich zu Literatursammlungen noch gering ist. Die Frage nach den für solche Retrodigitalisierungen beschreibbaren Möglichkeiten und Standards betrifft eine Reihe von technischen und lexikographischen Fragen, auf die im weiteren vor allem unter dem Blickwinkel der Benutzung digitaler historischer deutscher Wörterbücher kurz eingegangen werden soll.

Die Frage der technischen Standards der Retrodigitalisierung läßt sich knapp unter dem Blickwinkel betrachten, ob ein Text als Bildfolge von Buchseiten oder als Folge von Zeichen erfaßt wird. Die digitale Bildseite, das Image, liefert eine authentische Ansicht einer Buchseite des gescannten Exemplars einschließlich ggf. vorhandener farbiger Initialen, handschriftlicher Einträge, Stockflecken usw. Die digitalen Bildseiten lassen sich über Indizierungen nach Seiten, Kolumnentitel, Stichwörtern u. dgl. erschließen und bei der Benutzung aufrufen. Moderne Hochgeschwindigkeitsscanner erlauben bei der technischen Umsetzung des Printproduktes in die digitale Ebene ein sehr ökonomisches Vorgehen.

Die Image-Digitalisierungen bieten den Vorteil der authentischen Seitenwiedergabe, sie gestatten aber nicht, nach Wörtern bzw. Zeichenfolgen zu suchen. Solche Suchzugriffe machen es erforderlich, Zeichen für Zeichen des Printtextes zu erfassen und das Original in einer digitalen Volltextversion zu edieren. Dabei können Texterkennungsprogramme sehr wirksame Unterstützung bieten, wenn die Vorlage bestimmte Bedingungen erfüllt. Sauber gedruckte Antiquadrucke lassen sich so mit fast hundertprozentiger Fehlerfreiheit über solche OCR-Programme erfassen. Für Frakturdrucke oder Drucke mit häufig wechselnden Schriftarten und Schriftgrößen, wie dies in Wörterbüchern vielfach üblich ist, ist die automatische Zeichenerfassung noch sehr fehleranfällig und es führt z.Z. kaum ein Weg daran vorbei, die Texte abzuschreiben.

Das Abschreiben erweist sich dann als weitgehend problemlos, wenn die Texte auf dem normalen Zeichensatz beruhen und als Fließtexte gestaltet wurden. Unbeschadet dieser idealtypischen Situation ist grundsätzlich ein editorisches Verfahren nötig, wenn die typographische Gestaltung des Printtextes in die digitale Version übertragen werden soll. Die digitale Edition der Texte setzt vorrangig ein Kodierungssystem voraus, das eine zeichengenaue und layoutäquivalente Wiedergabe der Textoberfläche erlaubt. Ein entsprechend kodierter Text kann später unter Voraussetzung der erforderlichen Software für Folgeausgaben exakt reproduziert oder effizient überarbeitet werden. Sollen systematische inhaltliche Suchzugriffe auf Teile des digitalen Volltextes erschlossen werden, müssen die betreffenden inhaltlichen Ebenen oder Einheiten 
maschinenlesbar kodiert werden. Da Wörterbücher zahlreiche mikro- und makrostrukturelle Inhaltsebenen aufweisen, kann diese inhaltliche Auszeichnung u.U. eine sehr anspruchsvolle Aufgabe darstellen.

Die Textauszeichnung (text encoding oder text markup) kann technisch mit einer Auszeichnungssprache wie SGML (Standard Generalized Markup Language) erfolgen. Dadurch entsteht ein langfristig und plattformneutral nutzbares Textdatenformat. Das Grundelement einer markup-Sprache bildet die DTD (Document Type Defintion), die die gültige Grammatik für die Strukturierung eines Dokumentes enthält und alle Markierungen der Textelemente regelt. Die zur Verfügung stehenden wissenschaftlichen oder industriellen Standards können hier als durchaus entwickelt bezeichnet werden.

Retrodigitale Texte lassen sich netzgestützt oder als CD-ROM-Versionen für Benutzer erschließen. Als Repräsentanten der beiden skizzierten technischen Standards seien die Image-Versionen des Adelungschen Wörterbuchs, des Deutschen Wörterbuchs von H. Paul, des Mittelhochdeutschen Wörterbuchs von M. Lexer und des Deutschen Universalwörterbuchs der DudenRedaktion genannt. Die Neigung der Verlage, retrodigitale Wörterbuchtexte als CD-Versionen und nicht als Netzversionen anzubieten, ist im wesentlichen auf die Sorge vor unkontrollierter Weiterverwertung der Datenbestände zurückzuführen.

Zur Behandlung der technischen Zusammenhänge lexikographischer Retrodigitalisierungsansätze sei exemplarisch auf die Arbeit von Th. Gloning und Ch. Schlaps (1999) hingewiesen. Hier soll weiterführend der Frage möglicher Benutzungsbedingungen nachgegangen werden. Generalisierend kann zunächst dazu festgestellt werden, daß alle retrodigitalen Wörterbuchversionen den Bedürfnissen der dezentralen Literaturversorgung entsprechen. Diese Nutzungsbedingung ist unabhängig von der Art der Digitalisierung als Image oder Volltextversion. Mitunter kann die Imageversion sogar Vorteile bieten, die bei zeichengetreuer Wiedergabe nur schwer erreichbar sind. Die Imageversion des Adelungschen Wörterbuchs (Adelung digital) z.B. kann an beliebigen digital ausgestatteten Arbeitsplätzen benutzt werden, ohne daß Bibliotheksgänge anfallen, Kopierverbote zu beachten sind usw. Diese Version bietet zudem das seitengetreue Druckbild und damit eine Eigenschaft, die beim Umgang mit älteren Wörterbüchern besonders wünschenswert erscheinen kann.

Ein weiterer Aspekt der Literaturversorgung durch retrodigitale Wörterbücher ergibt sich aus der Hypertextverkettung verschiedener Werke zu einem Verbund. So kann z. B. das Duden Universalwörterbuch (Deutsches Universalwörterbuch digital) in der "PC-Bibliothek" des Brockhausverlages mit einer Reihe anderer Wörterbücher wie dem Rechtschreib-, Bild- oder Fremdwörterbuch im digitalen Verbund benutzt werden. Für die digitalen Versionen des Lexerschen Mittelhochdeutschen Wörterbuchs und des Mittelhochdeutschen Wörterbuchs von Benecke-Müller-Zarncke gilt entsprechendes (Mittelhochdeutsche Wörterbücher im Verbund). Damit wird es den Benutzern ermöglicht, 
verschiedene lexikographische Werke kombiniert am Bildschirm zu benutzen. Neben solchen bibliothekstechnischen Zusammenhängen ist die verlegerische bzw. bearbeitungsbezogene Nutzung der retrodigitalen Produkte anzusprechen. Die einmal retrodigitalisierte Volltextversion kann in der Weiterverwendung effizienter überarbeitet und für andere Wörterbuchvorhaben ausgewertet werden, als dies bei Printprodukten möglich ist. Eine neue Auflage kann auf der bestehenden Textbasis rascher erarbeitet werden. Auch wird es möglich, neue Wörterbücher aus vorhandenen zu gewinnen, indem Texte erweitert, selektiert oder aus verschiedenen Quellen zusammengeführt werden. Als dritte Ebene der Nutzung retrodigitaler Wörterbücher ist ihre systematische Auswertbarkeit in Form von Zeichenfolgensuchen zu nennen. Derartige Suchen sind in Printprodukten sehr umständlich und zeitraubend. Wer einmal alphabetische Printwörterbücher unter selektiven systematischen Gesichtspunkten z.B. bei der Ermittlung von Wortfeldern, Wortfamilien oder anderen Wortschatzausschnitten mit nichtalphabetischer Ausrichtung benutzt hat, weiß den grundsätzlichen Vorteil selbst einfacher digitaler Suchzugriffe zu schätzen. Hier liegt prinzipiell eine große Chance der digitalen Volltextversionen bei der Erleichterung und Effektivierung der Wörterbuchbenutzung.

Die skizzierten technischen Nutzungsverbesserungen haben zu einer verbreitet sehr optimistischen Einschätzung der künftigen retrodigitalen Wörterbuchwelt geführt. Dieser Optimismus verbindet sich mit Utopien u.a. über die Einbindung der Wörterbuchbenutzung in ein künftiges digitales Infotainment oder die Vernetzung des weitgehend retrodigitalisierten Printbuchbestandes (Storrer u.a. 1996).

Den zweifellos mit der Benutzung retrodigitaler Wörterbücher verbundenen Vorteilen sind jedoch aus der Kenntnis der Qualität der vorliegenden Wörterbücher einige grundsätzliche Nachdenklichkeiten gegenüberzustellen. Diese Nachdenklichkeiten sollen in ihrem Kern exemplarisch an einem Beispiel für eine offensichtlich gescheiterte systematische Nutzung einer retrodigitalen Wörterbuch-Volltextversion skizziert werden.

In einem Benutzungsexperiment wurde versucht, die wortgeschichtliche Markierung modern, die im Artikel ABBRECHEN des digitalen Paulschen Wörterbuchs (Paul digital) erscheint, als Ausgangspunkt für die Zusammenstellung aller Lemmata zu benutzen, in deren Beschreibung dieses Merkmal auftritt. Die Volltextsuche mit der betreffenden Zeichenfolge erbrachte 28 Treffer ohne flektierte Formen. Darunter befand sich das Stichwort MÓDERN und eine bunte Mischung objekt- und metasprachlicher Vorkommen einschließlich einer Form des dänischen Adjektivs in einer Quellenangabe im Artikel ENTWERFEN. Unstrittig liegen unter den Treffern auch Nachweise für die gesuchte wortgeschichtliche Markierung u.a. in den Artikeln STRAND, POTENZ, VERWARNEN usw. vor. Die Aufgabe des Benutzers besteht allerdings hier nicht nur in der Selektion des Suchbegriffs von Formalhomographen. In Kenntnis der Freiheiten der Beschreibungssprache der Wörterbücher wurde vorsorglich auch nach inhaltlich ähnlichen wortgeschichtlichen Markierungen für modern 
unter der Zeichenfolge heute gesucht. Unter den 2000 Treffern für die Zeichenfolge fanden sich zahlreiche wortgeschichtliche Klassifikationen, die synonymisch zu modern zu verstehen waren. Ähnlich, wenngleich vom Umfang her deutlich geringer war das Ergebnis mit dem Suchwort jünger. Der Versuch, einen mit diesem wortgeschichtlichen Merkmal bestimmten Lexikausschnitt systematisch auf der Grundlage der digitalen Volltextsuche zu ermitteln, ist, wie das Experiment zeigt, kaum gezielt und kontrolliert durchzuführen, weil die digitale Textstrukturierung unscharf ist und die zugrundeliegenden lexikographischen Informationen nur in der Zeichenebene durchsuchbar sind. Man kann sich ausmalen, wie ein Suchergebnis unter vergleichbaren Bedingungen aussähe, wenn die Suchform im Wortstamm flexivisch und orthographisch stark variiert erschiene. Für ein Verb wie reiten müßten historische Formen wie reytten, raiten, reuthen usw. alle separat ermittelt werden.

Man kann das skizzierte Ergebnis mit dem Hinweis auf die geradezu radikale Schlichtheit der digitalen Aufbereitungsform dieses Wörterbuchs relativieren. Die Ursache für die Resultate liegt auf der Hand. Da in der behandelten digitalen Version die Zeichenfolgen nur als ausdrucksseitige Einheiten, nicht als sprachliche Zeichen mit Inhalts- und Ausdrucksseite existieren, wird die Zeicheneinheit nur über die Ausdrucksseite konstituiert. Damit ein rechnergestützter Zugriff mit demselben Zeichenverständnis erfolgen könnte, wie es für eine herkömmliche Lesenutzung vorausgesetzt wird, müßte praktisch jede Zeichenfolge der retrodigitalen Version mindestens in ihrem Lemmawert kodiert werden.

Der editorische Standard des digitalen Paulschen Wörterbuchs beschränkt sich auf eine vereinfachte Erschließung der Textoberfläche des Originals. Inhaltlich und datenstrukturell sind nur Stichwörter und Artikel als Einheiten gekennzeichnet bzw. die Stichwortverweise mit Linkfunktionen unterlegt worden. Im interessierenden Bereich ist daher für die Benutzung nichts weiter zu erwarten als die Verknüpfung von Textwortformen und Stichwortformen. Für den wenig anspruchsvollen Benutzer mag dies deutlich mehr sein als nichts, für denjenigen, der z.B. den Robert Électronique kennt, ist dieses Mehr noch sehr nahe am Nichts.

Im Sinn der international erreichten digitalen Editionsstandards wäre zu erwarten, daß z.B. Mikro- und Makrostruktur der Artikel formal und inhaltlich angemessen ausgezeichnet und erschlossen würden. Dabei sind gestufte Lösungen denkbar. Mindestens aber wäre die Nutzung typographischer Textoberflächenmerkmale wie fett, mager, recte und kursiv als Hilfsindikatoren für Inhaltsebenen wie Objekt- und Metasprache zu fordern. Entsprechende Zuordnungen von Textformaten und inhaltlich bestimmten Textebenen sind programmunterstützt und damit automatisch möglich, außer natürlich bei Durchbrechungen des typographischen Systems. Weitergehende inhaltliche oder strukturelle Markierungen beträfen $u$. a. die Unterscheidung von Formalhomographen wie modérn und módern ebenso wie die Unterscheidung von 
Stichwörtern, Verweiswörtern, Belegwörtern, Verwendungsbeispielen, metasprachlichen Termini oder flexivisch-orthographischen Varianten der Wörter.

Im Vergleich zum Paulschen Wörterbuch zeigen sich bei jüngeren Retrodigitalisierungsansätzen im deutschsprachigen Raum Ergebnisse, die als Fortschritte zu interpretieren sind. Diese Einschätzung betrifft u.a. das retrodigitale Lexersche Wörterbuch, das im Verbund mit dem Mittelhochdeutschen Wörterbuch von Benecke-Müller-Zarncke und dem Findebuch netzgestützt zur Verfügung steht. Diese Netzversion bietet neben der Volltextsuche eine datenbankorientierte Abfragemöglichkeit auf den Ebenen Stichwort, grammatische Angabe, Siglen, Belegzitate, Etymologie und Bedeutungsangaben. Die z.Z. netzgestützt benutzbaren Datenbankebenen der Mittelhochdeutschen Wörterbücher weisen noch einige technische Einschränkungen auf, die hier jedoch vernachlässigt werden sollen. Der originale Wörterbuchtext kann zudem artikelweise im Zusammenhang gelesen und benutzt werden. Dabei sind alle Literatursiglen mit einem Quellenverzeichnis verlinkt. Über Links sind auch alle Stichwörter innerhalb des Verbundes vernetzt. Damit bietet diese Retrodigitalisierungsversion eine gegenüber der digitalen Version des Paulschen Wörterbuchs erheblich verbesserte technisch-inhaltliche Erschließung. Sie stellt auch eine modellhafte Lösung für Wörterbuchnetze dar.

Die Benutzung der Datenbankabfragen der Mittelhochdeutschen Wörterbücher im Verbund läßt sich an einigen Beispielen demonstrieren. Die Suche nach der Bedeutungsangabe "furchtsam" führt auf 15 Artikel, in denen ein gleichlautendes beschreibungssprachliches Element vorkommt. Diese Artikel können per Link unmittelbar von der Seite mit den Suchergebnissen angesteuert werden. Damit ergibt sich einerseits eine sehr komfortable Verbindung von Datenbank- und Artikelkomponenten, andererseits wird der ursprüngliche Charakter der Artikel als zusammenhängende Texte in wünschenswerter Form in die Benutzung eingebunden. Die Datenbankabfragen zeigen allerdings auch problematische Resultate. Weshalb das metasprachliche Element "Mark" zwar für den Artikel MARC stn. ausgewiesen wird, nicht jedoch für den Artikel HAGEMARCE "geschlossene, eingehegte mark", ist nicht ersichtlich. Möglicherweise kann man diese Erscheinung auf die von den Bearbeitern erwähnte noch nicht abgeschlossene Implementierung aller erforderlichen Softwarevoraussetzungen zurückführen.

Alle Abfragen beruhen offensichtlich auf einer 1:1-Zeichenfolgenabbildung. Der Benutzer kann pro Suchgang nur eine Textwortform angeben. Die Suche nach Feldweg in der Ebene der Bedeutungsangaben führt auf den Artikel VUOTERWEC, nicht aber auf den Artikel HAGELKRIUZE, in dem die beschreibungssprachliche Textwortform feldwegen auftritt. Dazu muß die Wortform feldwegen separat gesucht werden. Damit bietet diese retrodigitale Version zwar die Voraussetzungen für eine strukturierte Suche in verschiedenen Artikelebenen, sie macht es aber erforderlich, daß der Benutzer seine Suche gut konzipiert und alle Varianten des interessierenden Suchwortes selbst erzeugt, da eine Lemmatisierung nicht erfolgt ist. Da mittelhochdeutsche Wörterbücher, 
wie man annehmen darf, vorzugweise von einem engen Expertenkreis benutzt werden, kann man diese Benutzungsvoraussetzung ggf. tolerieren. Grundsätzlich aber stellt die fehlende Lemmatisierung der Textwortformen eine ganz erhebliche Benutzungsbarriere dar, weil die Konstruktion aller möglichen Flexions- und Schreibvarianten einen großen Arbeitsaufwand und im historischen Bereich sehr gute Sprachkenntnisse voraussetzt.

Für das Goethe-Wörterbuch ist ein retrodigitaler Prototyp (Gloning u.a. 1999) vorgestellt worden, der außer den Artikelgrenzen und den Stichwörtern ähnlich gestufte Zugriffe erlauben wird, wie sie für die Mittelhochdeutschen Wörterbücher skizziert worden sind. Als systematische Abfrageebenen sind Stichwörter, Bedeutungserläuterungen, Belege, Literaturnachweise und Synonymangaben vorgesehen. Ferner ist in diesem Prototypen die Vernetzung der Wörterbuchbelegstellen mit dem jeweiligen literarischen Werktext vorgesehen. Diese Vernetzung erscheint besonders attraktiv, weil sie den Beleg aus seiner lexikographischen Individuation wieder in den textuellen Zusammenhang bringt, ohne den er in vieler Hinsicht problematisch bleibt. Freilich läßt sich ahnen, daß eine über das Modell hinausgehende Umsetzung dieses nützlichen Plans vorläufig an verlagsrechtlichen Barrieren scheitern oder zu gebührenpflichtigen Lösungen führen wird. Ganz ähnlich sind Pläne zu beurteilen, die sich auf eine Vernetzung von Wörterbüchern und aktuellen Enzyklopädien beziehen.

Die Bearbeiter der retrodigitalen Goethe-Wörterbuchversion machen auch auf die Möglichkeit aufmerksam, verschiedene Ansichten der vorhandenen Artikel erzeugen zu können. So soll es möglich gemacht werden, sich z.B. nur die Bedeutungsstruktur eines Artikels unter Ausblendung aller anderen Textelemente zeigen zu lassen. Ferner wird angestrebt, die Belege ergänzend zur gedruckten Version nach verschiedenen Selektionsgesichtspunkten z.B. für den "minimalen Leser" oder einschließlich zusätzlichen Materials abzubilden.

Für das Deutsche Rechtswörterbuch wird ebenfalls z.Z. eine Retrodigitalisierung vorbereitet (Deutsches Rechtswörterbuch digital). Ein Prototyp ist netzgestützt für die Buchstaben I, N, O zugänglich. Das Startmenü bietet eine Buchstabenliste, über die man auf eine Stichwortliste und von dort auf die fensterweise aufgegliederte digitale Artikelfassung kommt. Für das Stichwort Igel wird zunächst die Wortklasse Maskulinum angegeben. Die im gedruckten Artikel unter den Gliederungsmarken I und II enthaltenen Positionen werden in zwei weiteren Fenstern als IGEL I und IGEL II überschrieben und mit den jeweiligen metasprachlichen Angaben versehen. Zu IGEL I findet sich die im Originalartikel enthaltene Erklärung "als zum Fang freigegebenes Tier". Ein zugeordnetes Unterfenster bietet als "Navigationshilfe" Links auf Belege und Lemmaliste. Klickt man den Link "Belege" an, öffnet sich ein weiteres Fenster mit der Beleg- und Fundstelle zu IGEL I, das untergliedert ist in je ein Feld für den Belegtext, die Datierung, eine bibliographische Sigle und eine Fundstellennummer. Die bibliographische Sigle ist als Link angelegt und führt auf die vollständige bibliographische Angabe. 
Während der Zugriff auf die verschiedenen Informationsschichten der Artikel in der digitalen Version des Rechtswörterbuchs prinzipiell den bisher beschriebenen Ansätzen zu vergleichen ist, wird durch die stark nach Fenstern segmentierte Information der Textzusammenhang der Artikel beeinträchtigt. Artikelübergreifende Recherchezugriffe sind z.Z. in dieser Version noch nicht möglich. Daher kann auch keine Aussage darüber gemacht werden, ob die für das Mittelhochdeutsche Wörterbuch kritisierte fehlende Lemmatisierung hier erfolgte. Positiv hervorzuheben ist in dieser Hinsicht jedoch die vom Ausgangsmenü anzusteuernde Schreibvariantenzuordnung, die die Schreibung ygel im Beleg des besprochenen Artikelabschnitts dem Lemma Igel zuordnet oder vom imbs auf Imbiß führt. Eine weitere Nutzungsmöglichkeit der digitalen Version des Deutschen Rechtswörterbuchs wird unter dem Teilmenü "Wortbelegung" geboten. Dort kann das nicht im Wörterbuch abgebildete Belegmaterial ab dem Alphabetabschnitt Mo- eingesehen werden.

Ein knapper retrodigitaler Ausschnitt aus M. Heynes Deutschem Wörterbuch (Heyne digital) ist abschließend zu nennen. Das Startmenü bietet unter der Rubrik "Index" in Submenüs verschieden strukturierte Zugriffe auf den Stichwortbestand. Zum einen kann die gesuchte Stichwortform nicht nur als ganzes Wort, sondern auch nach bestimmten Positionen in Komposita und auf Wortarten eingegrenzt gesucht werden. Zum anderen kann für die Ausgabe festgelegt werden, ob die Stichwortform mit Zusatzinformationen wie der Etymologie, den belegten Textwortformen und Verweiszielen angezeigt werden soll. Auf diese Weise können selektive Ansichten der Artikel gezeigt werden. Die Rubrik "Suche" erschließt den Weg zu einer Volltextsuche auf der Ebene nichtlemmatisierter Zeichenfolgen. Diese Suche kann auch selektiv nach bestimmten Textebenen wie Objekt- und Metasprache oder den Textwortformen zu den Stichwörtern gesteuert werden.

Gegenüber dem digitalen Paulschen Wörterbuch können solche Zugriffe technisch als durchaus entwickelt bezeichnet werden. Man wird Ch. Schlaps und Th. Gloning (1999) in der Beurteilung zustimmen, daß es trotz des erreichten Entwicklungsstandes zu den metalexikographischen Zukunftsaufgaben gehören wird, benutzerorientiert nach weiteren sinnvollen Erschließungsmöglichkeiten zu suchen, damit die hohen Investitionen in das erforderliche Markup für die Wörterbücher auch lohnen. Um aber einen solchen Kanon entwickeln zu können, muß nicht nur die Frage nach den gewünschten Benutzungsmöglichkeiten geklärt werden. Es muß auch geprüft werden, welche Standards in den Wörterbüchern vorliegen und von welchen Voraussetzungen Markup-Versuche ausgehen können. Dazu sollen im Anschluß einige Sachverhalte in historischen Wörterbüchern betrachtet werden. Darunter werden lexikographische Standardwerke gefaßt, die wie z. B. das Grimmsche Wörterbuch, das Deutsche Wörterbuch von H. Paul oder das Deutsche Wörterbuch von M. Heyne die deutsche Sprache in ihrer historischen Gesamtdimension beschreiben. 
Die lexikographische Texterstellung in diesen historischen Wörterbüchern zielt vielfach auf eine im Zusammenhang lesend entstehende Informationsentnahme durch die Benutzer, nicht darauf, standardisierte Informationen im systematischen Zusammenhang zu erschließen. Dabei wirken die von Vorstellungen des 19. Jahrhunderts geprägten lexikographischen Formen bzw. Benutzungsprojektionen bestimmend. So werden vor allem Textgestaltungsmöglichkeiten ausgeschöpft, die den Einzelartikel bzw. das Einzelwort anschaulich werden lassen. Die lexikographische Strukturkonsistenz ist ebenso wie die metasprachliche Konsequenz und die Ausführung von Vernetzungen meist nur ansatzweise entwickelt. Der Anteil frei umschriebener, elliptisch formulierter bzw. impliziter Information erweist sich als sehr hoch. Diskursive Tendenzen überlagern oder durchkreuzen die unterschiedlich entwickelten Gliederungsansätze.

Für die Textstrukturierung, die zur späteren systematischen digitalen Auswertung erforderlich ist, entstehen aufgrund solcher Textformen erhebliche Barrieren. Ein effizientes Markup, das über die automatische Kennzeichnung von typographischen Anfangs- und Endsignalen hinausgeht, ist kaum möglich. Eine solche automatische Auszeichnung der Texte wäre auch dann nicht mehr sinnvoll, wenn die typographischen Formate zwar als Indikatoren für inhaltliche Sachverhalte $\mathrm{zu}$ bestimmen sind, jedoch in einer diskursiven Verflechtung erscheinen, die bei einem selektierenden digitalen Zugriff zu weitgehend sinnlosen Textschnitzeln führte. Die häufig unscharfe mikrostrukturelle Gliederung der Artikel in den Printversionen kann nur dann für systematische digitale Suchen erschlossen werden, wenn zuvor eine Restrukturierung durchgeführt wird. Dabei muß der ursprüngliche Wörterbuchtext aufgrund kompetenzgestützter Interpretationen manuell ausgezeichnet werden. Dieser Arbeitsgang ist auch dann zwingend erforderlich, wenn typographische Formate im Text in der inhaltlichen Indizierungsfunktion ambivalent bleiben oder inkonsequent verwendet wurden. Wie die Untersuchungen von $\mathrm{K}$. Casemir zum Grimmschen Wörterbuch zeigen, ist das typographische System vom Ansatz her mehrdeutig und unterliegt zudem beträchtlichen Schwankungen. Der metasprachliche Beschreibungstext ist z.B. nicht nur regulär in kursiver Normalschrift ausgeführt, sondern erscheint u.a. auch recte gesperrt und kursiv gesperrt. Lemmavarianten werden in Versalien sowie - wie im übrigen auch die Belegtexte in Normalschrift - recte und kursiv ausgeführt usw. Eine automatische Umsetzung der typographischen Grenzensignale in Kodierungen des Markups müßte hier zwangsläufig zu einer beträchtlichen Ambivalenz der Zuordnung von typographischem Format und Inhaltsebene führen.

Die Defizite, die hier im lexikographisch-technischen Bereich erkennbar werden, lassen sich zweifellos mit einigem Aufwand durch Nachbearbeitung beheben. Dabei stellt sich naturgemäß die Frage nach der Relation von Aufwand und Ergebnis. Diese Frage stellt sich noch entschieden schärfer unter dem Blickwinkel der lexikographisch-inhaltlichen Qualität. 
In der bisherigen Diskussion um die Volltextretrodigitalisierung tritt die Beurteilung der inhaltlichen Wörterbuchqualität gegenüber dem Problem der Wörterbuchform und den datentechnischen Problemen weitgehend zurück. Offensichtlich geht man allgemein davon aus, daß die verfügbaren Werke konzeptionell und inhaltlich trotz des z.T. beträchtlichen Alters und bekannter Kritikpunkte so unstrittig sind, daß auch große finanzielle Investitionen gerechtfertigt erscheinen. Diesem Understatement entsprechen nicht alle Beobachtungen im hier berücksichtigten Spektrum.

Lexers Mittelhochdeutsches Wörterbuch oder das Grimmsche Wörterbuch in seiner abgeschlossenen Ausgabe werden seit langem als veraltet beschrieben (u.a. Schlaefer 1999: 94-95). Neue Werke oder Teilbearbeitungen sollen die alten Werke ersetzen. Dazu werden mit öffentlichen Mitteln erhebliche Aufwendungen gemacht. So wird für das Grimmsche Wörterbuch seit 1960 eine Neubearbeitung der Teile A-F durchgeführt. Für die Sprachstufe des Mittelhochdeutschen wurde unter Hervorhebung der Mängel der vorhandenen Wörterbücher (Mittelhochdeutsches Wörterbuch in der Diskussion 1988) durch die Göttinger Akademie der Wissenschaften (Jahrbuch 1998: 281) ein neues Langfristprojekt eingeleitet. Die kontinuierlichen Nachträge zum Lexerschen Wörterbuch einschließlich der Publikation des Findebuchs lassen ebenso wie Vergleiche der alten und der neuen Grimm-Ausgabe erkennen, daß in den alten Werken tatsächlich Lücken bestehen und aktuelle Forschungsinteressen nicht mehr adäquat befriedigt werden können. Dabei geht es offensichtlich nicht nur um Stichwortlücken, sondern auch um die Zusammensetzung der Quellenbasis, den Artikelaufbau und die Art der Wortbeschreibung.

Solche Mängel betreffen die Möglichkeiten, mit den retrodigitalen Wörterbuchversionen neue, vor allem systematische Recherchemöglichkeiten zu erschließen, in ganz erheblicher Weise, wie die folgenden Befunde veranschaulichen. Da Lexers Beschreibungssprache nicht nur sehr spontan und übersetzungsorientiert, sondern auch sehr auf Knappheit angelegt ist, sind weggelassene Bedeutungsangaben (Artikel WIDERSPRÜCHIG) oder referierende Verweise wie dasselbe (Artikel WIDERVEHTE) $i b$. (Artikel WIDERSPILN) ganz geläufig. Dazu kommen lateinische Interpretamente (Artikel WIDERSPRECHUNGE) als Bedeutungsangaben. In der beschriebenen Weise entsteht eine sehr uneinheitliche Informationsoberfläche, die zwar bei der Artikellektüre, nicht aber bei einem selektiven systematischen Zugriff kompensiert werden kann. Die oben skizzierte datenbankorientierte Suchabfrage für das Lexersche Wörterbuch findet damit nicht nur an der fehlenden Lemmatisierung der Textwörter ihre Grenze. Sie bleibt zwangsläufig defizitär, weil das verarbeitete Printprodukt schon Defizite und Inkonsequenzen aufweist. Die beste DTD kompensiert nicht das Fehlen der Bedeutungsangaben. Ganz analog zeigen sich beschreibungssprachliche Inkonsequenzen in anderen Wörterbüchern (Dummer u.a. 1998: 219). Hier scheint es geraten, die für retrodigitale Wörterbücher prophezeiten "neuen Nutzungsmöglichkeiten" (Mittelhochdeutsche Wörterbücher im Verbund) vor allem im Hinblick auf systematische Zugriffe 
mit großer Zurückhaltung zu beurteilen. Die neue, systematische Nutzung ersetzt im skizzierten Fall für wissenschaftliche Zielsetzungen keineswegs die traditionelle Lesenutzung der Artikel.

Die Aufwendungen für die Retrodigitalisierung erscheinen vor dem skizzierten Hintergrund nicht ohne weiteres schlüssig. Man wird aber das Argument zulassen müssen, daß es gegenwärtig keine besseren mittelhochdeutschen Wörterbücher gibt und daß das begonnene neue mittelhochdeutsche Wörterbuch voraussichtlich erst nach dreißig und mehr Jahren abgeschlossen zur Verfügung stehen wird. Für die heutige Forschergeneration und vermutlich auch noch für die folgende besteht durchaus ein Bedarf an digitaler Wörterbuchinformation, der mit dem vorgelegten Wörterbuchverbund provisorisch zu befriedigen ist. Hingegen kann die Begründung, die Forschungstradition müsse zugänglich gehalten werden, nur bedingt als Rechtfertigung für diese Art der aufwendigen Volltexterschließung betrachtet werden. Zum einen ist die Literaturversorgung mit den Printexemplaren der mittelhochdeutschen Wörterbücher durchweg zufriedenstellend, zum anderen könnten mit einer erheblich kostengünstigeren Image-Digitalisierung, die netzgestützt bereitgestellt wird, bestehende Literaturversorgungslücken geschlossen oder eine Dezentralisierung der Literaturversorgung rasch erreicht worden sein. Daß der retrodigitale Text der älteren mittelhochdeutschen Wörterbücher für eine künftige Überarbeitung oder Neuausgabe des Originals verwendet würde, scheint eine kaum realistische Perspektive.

Für den historischen neuhochdeutschen Bereich ergeben sich hinsichtlich der inhaltlichen Voraussetzungen für die Wörterbuchretrodigitalisierung ähnliche Sachverhalte wie bei den mittelhochdeutschen Wörterbüchern. Hier ist besonders die Rolle des Grimmschen Wörterbuchs zu beachten. Das Grimmsche Wörterbuch nimmt von der zweiten Hälfte des 19. Jahrhunderts bis in die Gegenwart eine dominante Rolle im schriftsprachlichen neuhochdeutschen Segment der Wörterbuchlandschaft ein. Diese Dominanz ist zwar durch die Entstehung des Frühneuhochdeutschen Wörterbuchs und eines entwickelten Spektrums von gegenwartssprachlichen Wörterbüchern jeweils an den Periodengrenzen bzw. durch das Deutsche Rechtswörterbuch und das Deutsche Fremdwörterbuch auf speziellen lexikalischen Ebenen in gewisser Weise eingeschränkt, jedoch nicht aufgehoben worden. Für den Kernbereich des historischen Neuhochdeutschen und den größten Teil des Alphabets steht damit ausschließlich die abgeschlossene Ausgabe des Grimmschen Wörterbuchs zur Verfügung.

Ganz fraglos stellt diese Ausgabe die umfangreichste Dokumentation zur historischen deutschen Lexik dar. An kritischen inhaltlichen und konzeptionellen Einschätzungen des Grimmschen Wörterbuchs fehlt es aber ebenso fraglos seit seiner Gründung nicht (vgl. Schlaefer 1999). Innerhalb dieser Einschätzungen kommt im gegebenen Zusammenhang den Stimmen ein besonderes Gewicht $\mathrm{zu}$, die monieren, daß die konzeptionellen und materiellen Grundlagen der Artikel oft kaum einzuschätzen sind und daß die Eigenwilligkeiten 
der Bearbeiter zu einer hochgradigen qualitativen Uneinheitlichkeit der Artikel geführt haben. Viele Artikel sind in ihrem Informationswert ohne die Aufarbeitung wörterbuchgeschichtlicher Hintergrundkenntnisse nur schwer oder gar nicht zu beurteilen. Die Erstellung einer wissenschaftlichen Ansprüchen genügenden retrodigitalen Volltextversion dieses Wörterbuchs steht damit vor deutlich größeren Problemen, als sie etwa für das Lexersche Wörterbuch beschreibbar sind. Zur Veranschaulichung derartiger Qualitätsprobleme sei exemplarisch auf einige Beobachtungen zum Artikel FINGER verwiesen.

Der von J. Grimm in der abgeschlossenen Ausgabe bearbeitete Artikel FINGER steht prototypisch für ein gelehrtes, aber unorganisiertes lexikographisches Arbeiten, das sich letztlich einer sinnvollen systematischen ErschlieBung entzieht. Unter dem Gliederungsabschnitt 1) (Deutsches Wörterbuch 3: 1650) werden Belege gebucht, die das Stichwort gar nicht enthalten, die weiteren 22 Gliederungsabschnitte mischen nach wenig nachvollziehbaren Gesichtspunkten Phraseologisches, Welt- und Völkerkundliches. Unter Gliederungsabschnitt 2) heißt es einleitend der ausgestreckte finger, zumal der rechten hand deutet, (...) gewinkt wird mit dem zeigefinger oder mit der ganzen hand, gedroht wird mit dem zeigefinger, der vierte finger heiszt der namenlose oder ungenannte (impudicus), finn. nimetöin sormi, und wenn auch den nordamerikanischen Mönilarris der dritte finger 'der ohne namen' genannt wird, ist es doch unser vierter, weil sie den daumen besonders rechnen und vom ersten finger zu zählen beginnen. (Deutsches Wörterbuch 3: 1651). Eine sprachgeschichtliche Information zum Stichwort Finger ist in diese Passage nur mühsam hineinzulesen. Und auch wenn es möglich wäre, in einer digitalen Version hier das Stichwort Zeigefinger zu finden, böte der Text doch auch dazu wenig sprachlich Relevantes. Die Hinweise auf die kulturhistorischen Implikationen der Fingergestik ist im Sprachwörterbuch ebenso überflüssig wie der Hinweis unter 3), der sich darauf bezieht, welche Fingerformen an Frauen als schön empfunden werden. Unter den Gliederungsabschnitten 5) und 6) des Artikels FINGER fehlt der Beschreibungstext ganz, dafür wird das Belegmaterial unter 13) um ein altirisches Zitat ergänzt. Die Quellenlage ist fast ausschließlich literarisch. Soweit es den neuhochdeutschen Zeitraum betrifft, sind schriftsprachliche schweizerische und österreichische Quellen weitgehend vernachlässigt worden.

Solche und ähnliche Befunde lassen sich auch in den anderen Teilen des Wörterbuchs machen. Dazu kommt in den späteren Teilen das Problem des völlig unübersichtlichen Artikelumfangs. Artikel wie GRUND oder STEHEN bedürften dringend der Neufassung und dies nicht nur, weil veraltete Textausgaben zitiert werden oder sekundäre Wörterbuchzitate 30\% der Belege ausmachten, sondern weil sie umfangsbedingt nicht mehr die Merkmale der Textsorte "Wörterbuchartikel" aufweisen. Hier würde auch die Möglichkeit, verschiedene Ansichten der Artikel wie z.B. die, nur Bedeutungsgliederung im Zusammenhang ausfiltern zu können, zu nichts führen, weil Abfolgen von "Bedeutungsangaben" wie unter STEHEN II, A, 7, b, v, bb) allenfalls noch in der diskursiven Textverflechtung einen Sinn machen. Daß solche Elemente 
ernsthaft in einer artikelübergreifenden datenbankorientierten Abfrage nach beschreibungssprachlichen Einheiten eine verwertbare Information ergäben, ist nicht ersichtlich. Unter der bezeichneten Stelle finden sich folgende durch Zählung einem Gliederungsabschnitt zugewiesene beschreibungssprachliche Angaben: bb) besonders häufig, cc) [ohne Beschreibungstext], dd) dafür auch, (...) gern in freiere gebrauchsweisen übergehend, o) [ohne Beschreibungstext], c) mannigfache adverbiale und präpositionale ausdrücke werden mit seite gebildet, $\alpha$ ) [ohne Beschreibungstext], $\beta$ ) [ohne Beschreibungstext], $\gamma$ ) mit näherer bestimmung im gen. (od. possessivpron.); so schon ahd. (Deutsches Wörterbuch 10, 2, 9: 1495-1496). Die vielen eingestreuten Einschübe mit ferner, auch, gewöhnlich, die vielfach eigene Gliederungsabschnitte konstituieren, wurden nicht berücksichtigt. Die retrodigitale Volltextversion wird hier nicht mehr als den zwingend im Zusammenhang zu lesenden Text erschließen können. Falls die Zeichenfolgen nicht lemmatisiert werden, ist auch auf die Belege für stehen eben nur dann ein Zugriff möglich, wenn der Benutzer weiß, daß er ston, stund, stand, st m,nd, stant, sten usw. suchen muß.

Andere Inhaltsprobleme der abgeschlossenen Ausgabe des Grimmschen Wörterbuchs können nur angedeutet werden. Auch in der retrodigitalen Version des Grimmschen Wörterbuchs bleibt die BLINDSCHLEICHE eine blinde giftige schlange, und unter dem Stichwort JUDE wird es weiterhin in dumpfem Antisemitismus lauten von ihren (der Juden) schlimmen eigenschaften werden namentlich ihre unreinlichkeit, sowie ihre gewinnsucht und ihr wuchersinn in mannigfachen wendungen betont. Für die 13 Stichwörter der Spalte 2391 in Band 4,2 von JUNGFRAUENMÄGDLEIN bis JUNGFRÄULEIN werden wie in vielen anderen Artikeln entweder gar keine oder nur kryptische Bedeutungsangaben stehen. SINKEISEN wird als Stichwort erscheinen, obwohl es nur niederdeutsch über einen Wörterbucheintrag nachgewiesen ist. Dafür werden auch in der retrodigitalen Version des DWB fast alle Fremdwörter fehlen und darüber hinaus alles, was nach dem jeweiligen, oft mehrere Generationen zurückliegenden Bearbeitungsschluß zu buchen gewesen wäre. Der Mängelkatalog könnte um zahlreiche Beobachtungen verlängert werden.

Keine noch so sorgfältige retrodigitale Aufbereitung wird diese Probleme beheben können. Die Frage nach Aufwand und Ergebnis einer Volltextdigitalisierung, die schon für die mittelhochdeutschen Wörterbücher gestellt worden ist, kann daher für das Grimmsche Wörterbuch kaum noch positiv beantwortet werden. Die Abfragen, die an digitale Volltextversionen auf den Ebenen der Stichwörter, der Objekt- oder Metasprache, der Etymologien, Belege und Quellennachweise gerichtet werden könnten, bleiben in weit höherem Maß, als es aus den angedeuteten Defekten des Lexerschen Wörterbuchs ersichtlich wurde, Stückwerk. Sie führen auf wenig Nützliches wie im Artikel Finger oder auf Abzulehnendes wie im Artikel Jude. Angesichts solcher Gegebenheiten fällt es schwer, sich vorzustellen, worin der mit einer Volltextversion erreichbare retrodigitale "Mehrwert" für die Wörterbuchbenutzer bestehen sollte. Die Tatsache, daß es für den fraglichen Objektbereich kein besseres Wörterbuch gibt 
und daß die Neubearbeitung des Grimmschen Wörterbuchs nur einen sehr beschränkten Buchstabenbereich revidiert anbietet, sollte weniger retrodigitale Bemühungen als lexikographische Neuansätze anspornen.

Retrodigitale Wörterbuchversionen, so ist zusammenfassend festzustellen, bieten in der Literaturversorgung und bei bestimmten systematischen Nutzungszugriffen zweifellos eine Verbesserung der bestehenden Möglichkeiten. Lexikographisch und inhaltlich sind die retrodigitalen Wörterbücher jedoch stets bestenfalls so gut wie ihre Printvorlagen. Die im einzelnen erreichbare retrodigitale Qualität hängt daher nicht nur von der Stringenz der DTD ab, sondern vor allem von der Qualität des verwendeten Printproduktes. Diese Qualität ist für einen Teil der Wörterbücher nachdrücklich in Frage zu stellen. Die retrodigitalen Produkte heben diese Mängel nicht auf, sondern tradieren sie. Systematische Abfragemöglichkeiten dürfen nicht darüber hinwegtäuschen, daß sie nur datentechnisch systematisch sind, inhaltlich jedoch eine vielfach lücken- und fehlerhafte Basis erschließen. Das Operieren mit dem Systematikbegriff bei retrodigitalen Wörterbüchern aus dem behandelten Spektrum sollte daher sehr zurückhaltend erfolgen, wenn nicht schwerwiegende Mißverständnisse in Kauf genommen werden sollen. Die inhaltlichen Mängel der historischen Lexikographie sind kaum durch Retrodigitalisierungen, verbreitet nicht einmal durch Überarbeitung, sondern nur mit zeitgemäßen lexikographischen Konzepten zu beheben. Diese Einsicht ist, wie die Argumentation für die Neubearbeitung des Grimmschen Wörterbuchs vor vierzig Jahren zeigt, nicht ganz neu und völlig unabhängig von der Entwicklung retrodigitaler Techniken.

\section{Literaturhinweise}

Adelung digital. http:/www.ub.uni-bielefeld.de/diglib/adelung/grammati/.

Adelung, J. Ch. 1808. Grammatisch-kritisches Wörterbuch der hochdeutschen Mundart, mit beständiger Vergleichung der übrigen Mundarten, besonders aber der Oberdeutschen, I-IV. Wien: Soltau.

Benecke, G. F., W. Müller und F. Zarncke. 1854-1861. Mittelhochdeutsches Wörterbuch, I-III. Leipzig: S. Hirzel.

Bergmann, R. (Hg.). 1998. Probleme der Textauswahl für einen elektronischen Thesaurus. Beiträge zum ersten Göttinger Arbeitsgespräch zur historischen deutschen Wortforschung, 1. und 2. November 1996. Hg. im Auftrag der Akademie der Wissenschaften in Göttingen v. R. Bergmann. Stuttgart: S. Hirzel.

Casemir, K. 2000. Zur Digitalisierung des Deutschen Wörterbuchs von Jacob Grimm und Wilhelm Grimm. Sprachwissenschaft 25: 77-102.

Deutsches Fremdwörterbuch. 1913 ff. Deutsches Fremdwörterbuch, I ff. Straßburg (Berlin u.a.): Trübner (de Gruyter).

Deutsches Rechtswörterbuch. 1914 ff. Wörterbuch der älteren deutschen Rechtssprache, I ff. Weimar: Böhlau.

Deutsches Rechtswörterbuch digital. http:/ /www.rzuser.uni-heidelberg.de/ cd2/drw/frameset.htm. Deutsches Wörterbuch digital. http://gaer27.uni-trier.de/GrimmWB/grimmwb.htm. 
Deutsches Wörterbuch. 1854-1971. Deutsches Wörterbuch von Jacob Grimm und Wilhelm Grimm, IXVI. Leipzig: S. Hirzel.

Deutsches Wörterbuch. Neubearbeitung. 1960 ff. Deutsches Wörterbuch von Jacob Grimm und Wilhelm Grimm. Neubearbeitung. Hg. v. der Berlin-Brandenburgischen Akademie der Wissenschaften und der Akademie der Wissenschaften zu Göttingen, I ff. Leipzig (/Stuttgart): S. Hirzel.

Deutsches Universalwörterbuch digital. Duden. Deutsches Universalwörterbuch. o. J. A-Z, 3. A. Version 1.1, PC-Bibliothek, Mannheim/Wien/Zürich: Dudenverlag.

Dummer, S., F. Michaelis, M. Schlaefer. 1998. Zur Digitalisierung historischer Wörterbücher. Lexikos 8: 1-29.

Findebuch. 1992. Findebuch zum mittelhochdeutschen Wortschatz. Mit einem rückläufigen Index. [Bearb. v.] K. Gärtner u.a. Stuttgart: S. Hirzel.

Gloning, Th. und Ch. Schlaps. 1999. Prototypen für ein elektronisches Goethe-Wörterbuch. Sprache und Datenverarbeitung. International Journal for Language Data Processing 2: 20-33.

Goethe-Wörterbuch. 1978 ff. Goethe-Wörterbuch. Hg. v. der Berlin-Brandenburgischen Akademie der Wissenschaften, der Akademie der Wissenschaften zu Göttingen und der Heidelberger Akademie der Wissenschaften, I ff, Stuttgart/Berlin: Kohlhammer.

Heyne digital. Heyne, M. 1890-1895. Deutsches Wörterbuch, I-III. Leipzig: Hirzel. http://Grimm. ADW-Goettingen.gwdg.de/heyne/hdw.html.

Heyne, M. 1890-1895. Deutsches Wörterbuch, I-III. Leipzig: Hirzel.

Jahrbuch 1998. Jahrbuch der Akademie der Wissenschaften zu Göttingen 1998 (1999). Göttingen: Vandenhoeck u. Ruprecht.

Lauer, B. (Hg.). 1999. Die Brüder Grimm und die Geisteswissenschaften heute. Ein wissenschaftliches Symposion der Brüder Grimm-Gesellschaft e. V. in der Paulinerkirche zu Göttingen am 21. und 22. November 1997. Schriften der Brüder Grimm-Gesellschaft, N.F. 30, Kassel: Brüder Grimm-Gesellschaft.

Lexer digital. Lexer, M. 1872-1878. Mittelhochdeutsches Handwörterbuch, I-III. Leipzig: S. Hirzel http://gaer27.uni-trier.de/MhdWB/.

Lexer, M. 1872-1878. Mittelhochdeutsches Handwörterbuch, I-III. Leipzig: S. Hirzel.

Mittelhochdeutsches Wörterbuch in der Diskussion. (1988). Symposion zur mittelhochdeutschen Lexikographie, Hamburg, Oktober 1985. Tübingen: Niemeyer.

Mittelhochdeutsche Wörterbücher im Verbund. o. J. Mittelhochdeutsche Wörterbücher im Verbund. Benecke/Müller/Zarncke — Matthias Lexer — Nachträge zum Lexer — Findebuch. Die wichtigsten lexikographischen Hilfsmittel für das Studium älterer deutscher Texte auf CDROM und im Internet.Entwickelt im Fach Germanistik der Universität Trier in Zusammenarbeit mit dem Kompetenzzentrum "Neue Publikationsformen für geisteswissenschaftliche Grundlagenwerke", in Verbindung mit der Akademie der Wissenschaften und der Literatur zu Mainz. (http://gaer27.uni-trier.de/MWV-online/MWV-online.html).

Paul, H. 1992. Deutsches Wörterbuch. Neubearb. A. v. H. Henne u. G. Objartel unter Mitarbeit v. H. Kämper-Jensen. Tübingen: Niemeyer.

Paul digital. Paul, H. 1992. Deutsches Wörterbuch. Neubearb. A. v. H. Henne u. G. Objartel unter Mitarbeit v. H. Kämper-Jensen. o.O., o.J.

Ris, R. 1998. Der schweizerische Anteil in den deutschen Großwörterbüchern. Bergmann, R. (Hg.). 1998: 113-124. 
Le Robert Électronique. 1994. Le Robert Électronique Dos-Macintosh-Windows (CD-Rom) Paris: Robert.

Schlaefer, M. 1999. Das Grimmsche Wörterbuch in der deutschen Wörterbuchlandschaft. Lauer, B. (Hg.) 1999: 93-124.

Storrer, A. und K. Freese. 1996. Wörterbücher im Internet. Deutsche Sprache 2: 97-153. 